Die Schweiz und die humanitäre Aktion

\title{
Strategie der Humanitären Aktion des IKRK gegenüber Konflikten des 21. Jahrhunderts
}

\section{Marion Harroff-Tavel}

\section{(2) OpenEdition}

1 Journals

Electronic version

URL: http://journals.openedition.org/sjep/632

DOI: 10.4000/sjep.632

ISSN: 1663-9677

Publisher

Institut de hautes études internationales et du développement

Printed version

Date of publication: 1 janvier 1999

Number of pages: 51-61

ISSN: 1660-5926

\section{Electronic reference}

Marion Harroff-Tavel, « Strategie der Humanitären Aktion des IKRK gegenüber Konflikten des 21.

Jahrhunderts ", Schweizerisches Jahrbuch für Entwicklungspolitik [Online], 18| 1999, Online erschienen am: 18 Juli 2012, abgerufen am 08 September 2020. URL : http://journals.openedition.org/sjep/632 DOI : https://doi.org/10.4000/sjep.632 


\title{
STRATEGIE DER HUMANITÄREN AKTION DES IKRK GEGENÜBER KONFLIKTEN DES 21. JAHRHUNDERTS
}

\author{
Marion HarRoff-TAVEL ${ }^{*}$
}

D as Internationale Komitee vom Roten Kreuz, das die Aufgabe hat, Leben und Würde der Opfer von Kriegen und innerstaatlicher Gewalt zu schützen und durch diese Situationen verursachte Leiden zu verhüten, geht auf eine Initiative von fünf Genfer Bürgern zurück. Die Gründung des IKRK in der Schweiz bildete den Anfang einer privilegierten Beziehung mit diesem Land - alle Mitglieder des Komitees sind Schweizer und werden im Kooptationsverfahren gewählt ${ }^{1}$. Die Beziehung wird durch die Grosszügigkeit der Schweiz hinsichtlich der Aktivitäten des IKRK geprägt, durch eine gewisse, einer gemeinsamen Kultur entsprungene Geistesgemeinschaft, aber auch durch die Sorge des Komitees, sich seine volle Unabhängigkeit zu bewahren. Dieser Wille des IKRK, die Selbständigkeit, die sein Merkmal ist, gegenüber allen Staaten zu wahren, und die Achtung dieses Willens durch die Schweizer Behörden haben in einem Sitzabkommen Ausdruck gefunden.

Die Wahl eines Artikels über das IKRK in einem Dossier, das der humanitären Aktion der Schweiz, dem Depositarstaat der Genfer Konventionen, gewidmet ist, und besonders das gewählte Thema - «Die Strategie der humanitären Aktion des IKRK gegenüber Konflikten des 21. Jahrhunderts» - sind somit leicht erklärlich. Der Augenblick ist umso günstiger, als das IKRK vor kurzem eine gründliche Reflexion über seine Zukunft abgeschlossen hat, an der das Komitee, die Kader sowie alle interessierten Mitarbeiter und Mitarbeiterinnen des Hauptsitzes und der Delegationen in den letzten zwei Jahren teilgenommen haben. Die Umsetzung des sich daraus ergebenden Aktionsplans soll im Jahr $2001 \mathrm{zu}$ Ende gehen, doch soll der eingeleitete Reflexionsprozess über diesen Zeitpunkt hinaus fortgesetzt werden.

Das vorgeschlagene Thema wird in drei Phasen behandelt: zuerst eine kurze Beschreibung des gestörten oder Konfliktumfelds, in dem die Aktion des IKRK erfolgt; danach die wichtigsten Herausforderungen, mit denen das IKRK konfrontiert ist; schliesslich die strategischen Ziele, die es sich gesetzt hat, um diesen Herausforderungen zu begegnen.

\section{ENTWICKLUNG DES KONFLIKTUMFELDS}

Von den aus dem Kalten Krieg oder der Entkolonisierung hervorgegangenen Konflikten bis zu den neuen Konflikten (Demokratische Republik Kongo,

* Politische Beraterin der Direktion des Internationalen Komitees vom Roten Kreuz, Genf.

1. Bugnion, François, La composition du Comité international de la Croix-Rouge, Revue internationale de la CroixRouge, juillet-août 1995, No. 814, pp. 473-493. Zum IKRK und zum internationalen Völkerrecht siehe auch Bugnion, François, Le Comité international de la Croix-Rouge et la protection des victimes de la guerre, Genève, Comité international de la Croix-Rouge, 1994, 1438 p. 
Albanien, Kosovo, usw.) - gleich ob es sich dabei um offene oder latente Konflikte handelt - an all diesen Auseinandersetzungen war das IKRK aus dem einen oder anderen Grunde zugunsten der Opfer beteiligt. Dies lässt einige Feststellungen zu.

\section{$\square$ Eine Palette verschiedenster Situationen}

Das IKRK sah sich im Laufe der letzten Jahre ganz unterschiedlichen Kriegen gegenübergestellt: zwischen dem «technologischen» Golfkrieg, welcher Konflikte mit raschen, gezielten Schlägen voraussehen lässt ${ }^{2}$, und dem Völkermordkrieg der Macheten in Ruanda gibt es wenig gemeinsame Punkte. Die technologischen Entwicklungen im Rüstungs- und Informationsbereich werden die Kriege, die von jenen geführt werden, welche Zugang zu dieser Technologie haben, von Grund auf verändern. Dabei ist nicht leicht vorauszusagen, wie die Antwort jener sein wird, die diese Technologie nicht besitzen, aber deren Zielscheibe sein werden: Terrorismus, Guerillakampf, psychologischer Krieg, Einsatz biologischer und chemischer Waffen? Nicht nur die Gefahren für die Zivilpersonen nehmen zu, sondern auch diejenigen, die ihnen zu Hilfe kommen, fallen immmer öfter Angriffen zum Ziel.

\section{$\square$ Langlebigkeit oder zyklischer Charakter der Konflikte}

Die Kriege, denen wir beiwohnen, sind von langer Dauer. Wenn ein Waffenstillstand oder ein Friedensabkommen unterzeichnet wird, so bedeutet dies häufig nicht mehr das Schweigen der Waffen und noch weniger das Eintreten zu einer «normalen» Situation, welche die Rückkehr der Vertriebenen, den freien Personenverkehr, die Demoblilisierung der Streitkräfte und ihre Wiedereingliederung ins Zivilleben, die Möglichkeit, das Schicksal der Verschollenen aufzuklären, ja sogar die Entwicklung von Beziehungen zwischen ehemaligen Feinden erlaubt. Die Abkommen von Dayton, Oslo oder Lusaka zeugen davon. Andere Konflikte, wie der in Tadschikistan, tauchen periodisch wieder auf, je nach dem Auf und $\mathrm{Ab}$ der Verhandlungen zur Beilegung des Streitfalls, den Provokationen kriegerischer Fraktionen oder dem Ablauf der Jahreszeiten.

Vielleicht richten sich einige Regionen der Welt, namentlich unter dem Einfluss der Globalisierung, in einer Situation «dauerhafter Unordnung» ein, die an die Zeit des Mittelalters erinnert. Fest steht jedenfalls, dass die Wende, welche die derzeitigen Konflikte mit dem Auftreten neuer Mächte, einer Form unvollendeter Auflösung des Staates, dem zunehmenden Gewicht von Parallelwirtschaften und Privatinteressen annehmen, die Gedankenschemata herausfordert, von denen sich das IKRK jahrzehntelang leiten liess ${ }^{3}$. Die Kriegserklärungen, Anerkennungen kriegsführender Parteien und Friedensverträge, die dem Krieg wirklich ein Ende setzen, erscheinen in einigen Gebieten der Erde zumindest als überholt.

2. «La version futuriste du Blitzkrieg», nach Thérèse Delpech in La guerre parfaite, Flammarion, 1998, p. 31. Vgl. auch Ramonet, Ignacio, Géopolitique du chaos, Paris, Editions Galilée, 1997, pp. 111-124 (Abschnitt zum Thema «rébellions à venir»).

3. Cerny, Philip G., Neomedievalism, Civil War and the new Security Dilemma: Globalisation as Durable Disorder, Civil Wars, Band 1, Nummer 1, Ilford, Frank Cass \& Co. Ltd., Frühjahr 1998, S. 36-64. Siehe auch die Konfliktanalyse in Duffield, Mark, Aid policy and post modern conflict: A critical Review, Occasional Paper 19, The University of Birmingham, School of Public Policy, 1998, 104 p. 


\section{$\square$ Die Schwierigkeit, Konfliktopfer von Verarmungsopfern zu unterscheiden}

Die Opfer von Konflikten sind lange Zeit als Verwundete, Gefangene, Vertriebene oder Flüchtlinge, an ihrer körperlichen oder geistigen Unversehrtheit beinträchtigte oder durch Plünderung all ihrer Habe beraubte Personen definiert worden. Doch können die Auswirkungen einer durch den Krieg verschlimmerten Wirtschaftskrise genau so furchtbar sein. Waren die Greise in Armenien, Anfang der neunziger Jahre - ohne Mittel zu heizen, bei einer Temperatur von -10 Grad, wegen einer Energiekrise, die mit der Auflösung der Sowjetunion zusammenhing, aber durch die regelmässige Sprengung von Ölleitungen in Verbindung mit dem Karabach-Konflikt noch verschlimmert wurde - waren sie Konfliktopfer, um die sich das IKRK kümmern musste, oder nicht? In den Ländern, in denen die Verarmung Todesopfer fordert, wird es schwierig, zwischen den Opfern zu unterscheiden.

\section{VOM IKRK ZU ÜBERWINDENDE HERAUSFORDERUNGEN}

Die Aktion des IKRK wird von den tatsächlichen und den rechtlichen Behörden nicht immer als willkommen erachtet, selbst wenn die Konfliktopfer sie bitter nötig haben. Die Gründe hierfür sind vielfältig: Wahrnehmung der humanitären Aktion als Einmischung in die inneren Angelegenheiten des Staates; eine Art übersteigerter Stolz, der dazu führt, jede Hilfe von aussen abzulehnen, ja ihren Nutzen zu leugnen, um sich von jeglicher Vormundschaft zu befreien; der Wille, unter Ausschluss der Öffentlichkeit Operationen zur Ausrottung der Bevölkerung durchzuführen oder Terror anzuwenden, um die Aufsässigen in die Flucht zu jagen; die Ablehnung des Ausländers, bzw. desjenigen, der als Spion angesehen wird; die Furcht vor einer Verbreitung freizügiger «westlicher» Ideen durch die expatriierten Delegierten, die in einem geschlossenen Umfeld umstürzlerisch wirken könnten, oder auch die im sozialen bzw. wirtschaftlichen Bereich destabilisierende Wirkung des Eindringens einer als «reich» angesehenen Organisation. Der Ehrlichkeit halber muss man sagen, dass das Problem nicht neu ist, auch wenn es einen akuteren Charakter angenommen hat. Nachfolgend sollen die Herausforderungen von heute ausführlicher erläutert werden.

\section{$\checkmark$ Verletzlichkeit der humanitären Aktion}

Einer der Faktoren für die Verletzlichkeit der humanitären Aktion ist ihre Sichtbarkeit. Sie tritt an vielen Kriegsschauplätzen klar zu Tage. So blieb das IKRK in Afghanistan mit seinen einigen hundert expatriierten Delegierten und nationalen Mitarbeitern, die zu Jahresbeginn dort arbeiteten, nicht unbemerkt. Mit der Abreise der NRO ist das IKRK vorübergehend zum wichtigsten humanitären Akteur geworden. In Gebieten wie Abchasien, die man in zwei bis drei Autostunden durchfahren kann, bilden zehn Delegierte eine bedeutende ausländische Vertretung. Das IKRK ist somit zuweilen ein Fenster zur internationalen Bühne, der einzige oder wichtigste Gesprächspartner. In einigen Fällen ist dies ein Schutz. In anderen Fällen ist es eine Gefahr: wer immer einer ausländischen Präsenz schaden will, die internationale Gemeinschaft davon überzeugen will, dass die Region instabil ist, ganz offenkundig für seine Sache werben will, die öffentliche Meinung erschüttern will, der hat dort eine gefundene Zielscheibe - eine 
Zielscheibe, die, obwohl sie sich aus jeder politischen Kontroverse heraus-hält, als mit einem politischen Gewicht behaftet wahrgenommen wird. Aber die Sichtbarkeit des IKRK ist nicht allein im Spiel. Alle oben angeführten Faktoren, die zur Ablehnung seiner Aktion führen, machen das IKRK verletzlich.

\section{$\square$ Das in Frage gestellte humanitäre Völkerrecht}

Ein Grossteil der Aktivitäten des IKRK gründen sich auf das bei einem bewaffneten Konflikt zur Anwendung kommende humanitäre Völkerrecht, das sich sowohl mit der Führung der Kampfhandlungen als auch mit der Behandlung der Verwundeten, Kranken, Gefangenen und Schiffbrüchigen sowie der Zivilbevölkerung befasst. Wenn ein Konflikt ausbricht, ruft das IKRK allen kriegsführenden Parteien vorbeugend ihre Verpflichtungen als Vertragsparteien der Genfer Konventionen in Erinnerung. So unterbreitete das IKRK beispielsweise Ende August 1998 der Demokratischen Republik Kongo und den beteiligten Drittstaaten sowie dem Rassemblement Démocratique du Congo ein Memorandum über die Anwendbarkeit des humanitären Völkerrechts. Wenn das humanitäre Recht verletzt wird, protestiert das IKRK bei den Behörden, indem es diese Regeln anführt. Gewiss gibt es Ausnahmen, wenn das IKRK der Ansicht ist, dass die Tatsache, eine Situation öffentlich als Konfliktsituation zu bezeichnen, seine Tätigkeiten zugunsten der Opfer gefährden könnte. Viele Regierungen ziehen es vor, wenn erklärt wird, die öffentliche Ordnung werde durch kriminelle Handlungen von Terroristen, statt durch einen Machtwiderstand organisierter Kämpfer, gestört. Das Gegenteil zu behaupten, öffnet nicht unbedingt Türen. Im grossen und ganzen setzt sich das IKRK, das der Initiator des humanitären Völkerrechts ist, jedoch mutig für dieses Recht ein.

\section{Das humanitäre Völkerrecht}

Das humanitäre Völkerrecht ist ein Komplex von Regeln, die in Zeiten bewaffneter Konflikte darauf abzielen, einerseits die Personen zu schützen, die nicht oder nicht mehr an den Kampfhandlungen teilnehmen, andererseits die Methoden und Mittel der Kriegsführung zu beschränken. Die vier Genfer Konventionen von 1949 und ihre beiden Zusatzprotokolle von 1977 sind die wichtigsten Rechtsinstrumente des humanitären Völkerrechts, welches auch "Recht der bewaffneten Konflikte" oder "Kriegsrecht " genannt wird. Unter den wesentlichen Regeln dieser Instrumente lassen sich folgende erwähnen: das Verbot, einen Gegner, der sich ergibt oder kampfunfähig ist, zu töten oder zu verwunden; die Verpflichtung, die Verwundeten und Kranken aufzunehmen und zu pflegen; die Achtung des Lebens und der Würde der gefangen genommenen Kämpfer und der Zivilbevölkerung, die sich in der Gewalt der gegnerischen Partei befindet, sowie die Verpflichtung, Angriffe nur gegen militärische Ziele zu richten.

Als Initiator des humanitären Völkerrechts trägt das IKRK zur Entwicklung dieses Rechts bei und fördert seine Einhaltung. Dies erreicht es auf verschiedene Art und Weise: durch seine Aktion zugunsten der Opfer bewaffneter Konflikte, durch seine Programme zur Verbreitung des humanitären Völkerrechts, durch ein gezieltes Vorgehen bei den verantwortlichen Behörden, wenn das Recht verletzt wird, und durch die Bereitstellung eines Rechtsgutachtens auf dem Gebiet der Verfolgung von Verstössen gegen die Genfer Konventionen.

Das humanitäre Völkerrecht ist indes zur Zeit einigen Attacken ausgesetzt. Zunächst sehen einige in ihm einen Ausdruck des Westens wohingegen 188 Staaten den Genfer Konventionen angehören, deren Zusatzprotokolle nach der Entkolonisierung ausgearbeitet wurden. Das IKRK kann aber dieser Auffassung 
gegenüber nicht gleichgültig bleiben, sei sie nun begründet oder nicht. Zum anderen lähmt der Zusammenbruch der staatlichen Strukturen die Anwendung des humanitären Völkerrechts durch die Streitkräfte, sobald die Befehlsketten verschwimmen. Zudem können bei innerstaatlichen Konflikten gewisse Gewaltakteure, die vom Konflikt profitieren, um durch Handlungen rein individuellen Banditentums zu töten und zu plündern, nicht als « Konfliktparteien », mit allen Verpflichtungen, die das rechtlich mit sich bringt, bezeichnet werden. Als erschwerender Faktor kommt hinzu, dass man sich erneut auf das Konzept des «gerechten Krieges» beruft. Die Tatsache, Zivilpersonen, die einer Ethnie angehören, die für Massaker verantwortlich gemacht wird, über Tausende von Kilometern zu verfolgen, ist für einige eine gerechte Sache. Der Begriff der Schuld einer Bevölkerungsgruppe taucht wieder auf, wobei er Rache oder Kollektivstrafen rechtfertigt und die Grundlagen des humanitären Völkerrechts, das dem Individuum Schutz gewährt, als solche in Frage stellt. Schliesslich hat die Straflosigkeit, derer sich lange Zeit jene erfreut haben, welche die schlimmsten Übergriffe begingen, die Geltung dieses Rechts geschwächt ${ }^{4}$.

\section{$\square$ Menschlichkeit, Unparteilichkeit, Neutralität: drei schlecht verstandene Grundsätze ${ }^{5}$}

Zunächst die Menschlichkeit. Der Leser und die Leserin werden wahrscheinlich erstaunt sein, dass dieser Grundsatz Probleme aufwerfen kann. Doch ist das Mitgefühl mit dem gefallenen Feind nicht überall verbreitet. Viele Gesprächspartner des IKRK verstehen nicht, dass das Komitee Menschen zu Hilfe kommen möchte, die als Verbrecher oder Terroristen angesehen werden. Die Überzeugung, dass in jedem menschlichen Wesen eine gewisse Würde steckt, wird nicht von allen geteilt. Im übrigen geben einige Zivilisationen, besonders in Asien, dem Interesse der Gemeinschaft den Vorrang vor dem Interesse des Individuums, das zuweilen dem Gemeinwohl geopfert werden muss. Menschlichkeit wird als eine Geste verstanden, die nur Sinn hat, wenn sie der grossen Mehrheit zugute kommt.

Die Unparteilichkeit des IKRK schreibt ihm vor, bei seinen Aktionen keinerlei auf politische, religiöse, ethnische oder sonstige Zugehörigkeit gegründete Diskriminierung vorzunehmen, aber auch prioritär denjenigen zu helfen, die sich in grösster Not befinden. Jedem nach seinen Bedürfnissen. In den Ländern Osteuropas und Zentralasiens zum Beispiel wird dieses Prinzip nicht immer verstanden. Die Dorfältesten sammeln die an Betagte, Behinderte und Mütter kinderreicher Familien verteilten Nahrungsmittelpakete wieder ein und teilen sie unter allen Dorfbewohnern auf, einschliesslich jenen, die mehr Mittel als die anderen haben und eine Rente beziehen. Es gibt auch Gesellschaften, wo die Männer, die das Überleben des Stammes sichern, zuerst bedient werden. Schliesslich ist es mitunter für die Bewohner eines armen Gebiets unverständlich, dass reichlich Hilfe für Vertriebene fliesst, die sie nicht immer mit Begeisterung aufnehmen. Das IKRK muss für dieses Empfinden, das in Feindseligkeit umschlagen kann, empfänglich sein und zuweilen gegen das Unparteilichkeitsprinzip verstossen.

4. Die Annahme des Statuts des Internationalen Strafgerichtshofs am 17. Juli 1998 in Rom sollte jedoch dazu beitragen, dass bestimmte mutmassliche Urheber von Kriegsverbrechen vor Gericht gestellt werden.

5. Die Grundsätze der Internationalen Bewegung vom Roten Kreuz und Roten Halbmond sind in der Präambel der Statuten der Bewegung aufgeführt, die von der XXV. Internationalen Rotkreuzkonferenz im Oktober 1986 in Genf angenommen wurden. 
Was die Neutralität betrifft, so wird sie oft als eine Form stillschweigender Duldung oder einen Mangel an Mut ausgelegt. Wie soll man dem Minister eines Landes, dessen Gebiet zu einem Fünftel besetzt ist und das ums Überleben von Hunderttausenden von Vertriebenen kämpft, erklären, dass das IKRK diese Besetzung nicht verurteilen wird, weil ihm sonst die Türen zum Angreifer verschlossen würden, der Gefangene hält und die Zivilbevölkerung, die bei der Besetzung nicht geflohen ist, misshandelt? Für den besagten Minister hätte eine Verurteilung durch eine international geachtete Organisation oft mehr Bedeutung als jede Schutzaktion, auch für die Seinigen. Es kann nur erneut festgestellt werden, dass die Neutralität für das IKRK ein Mittel ist, um Zugang zu den Opfern zu erhalten, und dass sie nicht aus Schweigen besteht. Das IKRK führt einen beharrlichen vertraulichen Dialog mit den Behörden im Fall der Verletzung des humanitären Völkerrechts und behält sich vor, bei anhaltender, schwerwiegender und vorsätzlicher Verletzung seine Stimme zu erheben.

\section{$\square$ Der humanitäre «Wettbewerb»}

Wenn Dutzende humanitärer Organisationen, von denen jede eigene Ziele hat (nur wenige haben, wie das IKRK und das UNHCR, ein Mandat der internationalen Gemeinschaft), an einem Kriegsschauplatz zusammenströmen, behaupten, denselben Opfern zu helfen - denjenigen, über deren Leiden in den Medien am meisten berichtet wird und für die die Gelder fliessen - und dies aus sehr unterschiedlichen Gründen tun, so ist es die erste, verständliche Sorge der Behörden, die Lage zu meistern. Gewiss leisten viele dieser Organisationen mit grossem Einsatz eine aussergewöhnliche Arbeit, und diese Solidaritätsreaktion kommt den Opfern zugute. Sie schafft aber auch Probleme: Doppelspurigkeit der Aktivitäten auf Kosten vergessener Opfer, negative Auswirkungen der Verfehlungen einiger humanitärer Organisationen auf alle anderen, Schwierigkeit für das IKRK, seine Spezifizität als neutraler Vermittler verständlich zu machen, der von den Staaten ernannt wurde, um bestimmte in den Genfer Abkommen aufgeführte Aufgaben zu erfüllen.

Die Herausforderung ist zweifacher Art: zu erreichen, dass die humanitäre Aktion insgesamt wirkungsvoll ist und allen Bedürftigen zugutekommt, und in die humanitäre Botschaft die Verpflichtung der Staaten aufzunehmen, die politischen Probleme, die so viel Leid mit sich bringen, an der Wurzel zu behandeln, bzw. sie zu verhüten, da die humanitäre Aktion kein Alibi sein darf.

\section{DIE STRATEGISCHEN ZIELE DER HUMANITÄREN AKTION DES IKRK}

$\mathrm{Zu}$ Beginn der Reflexion über seine Zukunft stand das IKRK vor grundlegenden Entscheidungen: Wollte und konnte es seine Aktion auf Situationen schwerer Gewalt, auf Menschen in Not, bei denen niemand den Charakter von Konfliktopfern bestreiten konnte, auf lebenswichtige Interessen konzentrieren, oder sollte es auf der Grundlage einer umfassenderen Vision, den Konflikten vorund nachgeschaltete Aktivitäten entfalten und die Palette seiner Tätigkeiten erweitern? Wollte es zwischen sich und den übrigen humanitären Akteuren, einschliesslich der Nationalen Rotkreuz- und Rothalbmondgesellschaften, eine klare Distanz setzen, um seine Spezifizität zu wahren, oder sollte es eine breite 
Zusammenarbeit, ja sogar in bestimmten Fällen die Verantwortung für eine Koordination akzeptieren, nach der es aus eigenem Antrieb schwerlich trachtete? War es bereit, sich mehr für die Menschenrechte einzusetzen und Partner bei der Wahrung eines humanitären Rechts zu mobilisieren, über das es lange Zeit eifersüchtig gewacht hatte? Konnte es akzeptieren, sich mit einem beliebigen Gewaltakteur wie mit Staaten zu unterhalten, einschliesslich Wegelagerern oder Schiebern, deren Kriminalität legitimerweise als gemeinrechtlich angesehen werden konnte, die sich aber zu unumgänglichen Akteuren der Konflikte verwandelten? Diese Fragen waren, unter anderen, wesentlich.

Die Wahl ist nicht leicht gefallen. Unterschiedliche Standpunkte wurden, zuweilen leidenschaftlich, vorgetragen. Entscheidungen wurden getroffen. Sie wurden von einer grundlegenden Reflexion vorgegeben, von einer Praxis, die sich beträchtlich weiterentwickelt hatte - aber vielleicht musste man von den Fakten zu einer Kodifikation übergehen - von einem schmerzhaften Bewusstwerden der Verletzlichkeit der Institution gegenüber Angriffen und vom Willen zur Veränderung. Der Zeitpunkt ist gekommen, diese Entscheidungen vorzustellen, wobei man sich einige Freiheiten gegenüber der offiziellen Präsentation, die das IKRK von den Ergebnissen seiner Reflexionen gegeben hat, herausnimmt.

\section{Ein genaues Verständnis vom Konfliktumfeld und seinen möglichen Entwicklungen erlangen}

Es geht darum, die Konflikte vorhersehen zu können, indem man die Fähigkeit hat, die Kenntnisse von Experten oder in der Konfliktforschung spezialisierten Zentren sowie die von den IKRK-Delegationen «im Feld» gemachten Beobachtungen zu analysieren. Wenn das Komitee auch nicht den Ausbruch eines bewaffneten Konflikts im Nahen Osten im Jahre 1973 vorausgesehen hat, so hatte es sich hingegen zwei Jahre vor Ausbruch des Tschetschenien-Konflikts im Nordkaukasus niedergelassen, was es ihm erlaubte, sofort zur Tat zu schreiten. In Gewaltsituationen, welche Opfer fordern, muss man auch ein Netz von Kontakten in den verschlossensten Kreisen entwickeln, vom Leuchtenden Pfad in Peru bis zum LTTE in Sri Lanka, über die paramilitärischen Kreise in Kolumbien, ohne die Zivilgesellschaft zu übersehen. Dies ermöglicht es, die Machtzentren zu identifizieren, die als Hebel für die Durchsetzung des humanitären Rechts dienen können und eine Schlüsselrolle im Sicherheitsbereich haben. Schliesslich und vor allem muss man den Opfern nahe bleiben und sie anhören, indem man für die soziokulturelle Umwelt empfänglich ist, in der sie leben - was das IKRK zur Zeit tut, indem es diese anlässlich des 50. Jahrestages der Unterzeichnung der Genfer Konventionen von 1949 mittels Fragebögen und Gesprächen konsultiert.

Diese Verwurzelung des IKRK im Konfliktumfeld wird erheblich gefördert durch seine nationalen Mitarbeiter und seine Kollegen von Nationalen Gesellschaften, die sich zuweilen allein in Weltgegenden befinden, die den expatriierten Delegierten verschlossen sind. In einigen Gebieten Somalias und Tschetscheniens konnte durch ferngesteuerte Programme eine Präsenz bei den Opfern aufrechterhalten werden, denen man andernfalls nicht hätte helfen können. 
Das IKRK kann zu jeder Zeit humanitäre Tätigkeiten entfalten, doch ändert sich ihre Art je nach der Situation. Seine Regionaldelegationen in Washington, Bangkok, Buenos Aires oder Taschkent widmen sich der Aufgabe, das humanitäre Völkerrecht bekannt zu machen, von den Staaten zu erreichen, dass sie ihre nationale Gesetzgebung anpassen, um sie mit dem humanitären Völkerrecht in Übereinstimmung zu bringen, und die operationelle Kapazität der Nationalen Rotkreuz- und Rothalbmondgesellschaften im Fall eines Konflikts zu verstärken. Sobald die öffentliche Ordnung jedoch durch repressive Massnahmen (Verhaftungen, Diskriminierungsmassnahmen, psychologische Belästigung usw.), bzw. in extremeren Fällen durch Aufstände, einzelne und sporadische Gewalttaten, Massenverhaftungen, Zwangsverschleppungen oder Hinrichtungen ohne Gerichtsverfahren gestört wird, kann das IKRK in seiner Eigenschaft als spezifisch neutrale und unabhängige Institution seine humanitären Dienste anbieten. Es konzentriert sich dann auf die Bedürfnisse von Risikogruppen, namentlich Gefangene. Wenn ein Konflikt ausbricht, führt es seine traditionellen Aufgaben aus. Die Einfrierung des Konflikts durch ein Abkommen oder einen Waffenstillstand hat keinen Rückzug des IKRK zur Folge, vor allem, wenn das Komitee der Meinung ist, dass der Streitfall nicht beigelegt ist, dass die Kampfhandlungen wieder aufleben können und dass die humanitären Probleme weiter bestehen (keine Massenrückkehr von Vertriebenen, erforderlicher Schutz für die Gefangenen, Schicksal der Vermissten nicht aufgeklärt, Identifikation der Toten vorzunehmen, usw.). Jedoch zieht sich das IKRK in einer postkonfliktuellen Phase aus den Hilfseinsätzen zurück, wenn der Wiederaufbau vorangeht und eine Friedenslogik eingekehrt ist.

Mit einem Wort sieht das IKRK seine Rolle nicht auf die eines Feuerwehrmanns beschränkt, der mit seinem Spritzrohr erst angelaufen kommt, wenn das Haus bereits in Flammen steht, und dann wieder abzieht, auch wenn die Gefahr besteht, dass die schwelende Glut das Feuer wieder anfacht. Dies wäre viel zu gefährlich. Es wäre kostspielig, denn der Aufbau einer angepassten Logistik (Fahrzeugflotte, Häuser, Ausrüstung) erfordert Energie und Mittel. Schliesslich würde dies auch den Verlust eines wertvollen Netzes an Gesprächspartnern bedeuten. Deshalb setzt das IKRK auf eine dauerhaft angelegte Aktion und auf die Komplementarität zu den anderen Komponenten der Internationalen Rotkreuz- und Rothalbmondbewegung, nämlich die Nationalen Rotkreuz- und Rothalbmondgesellschaften und ihre Föderation.

\section{$\square$ Eine globale Lösung für die Bedürfnisse entwickeln}

In Situationen offener Gewalt (Konflikte, innere Unruhen) beabsichtigt das IKRK, eine globale Evaluation der Bedürfnisse vorzunehmen, einschliesslich der Bedürfnisse jener Personen, die durch Funktionsstörungen bzw. durch den Ausfall von unentbehrlichen Dienstleistungen oder Gütern betroffen sind, auf die sie Anspruch hatten oder an die sie gewöhnt waren. Dabei kann es sich um die gesamte Bevölkerung von Ländern oder Gebieten handeln, die der Gewalt zum Opfer gefallen sind, oder auch um bereits vorher anfällige Gruppen (mangelhafte Wasserversorgungssysteme, Medikamentenmangel, in Not geratene Altersheime). Das IKRK hat sich jedoch zum Ziel gesetzt, sich darauf zu beschränken, auf Überlebensprobleme und längerfristig auf Probleme eines 
Lebensunterhalts in Würde einzugehen (zum Beispiel durch landwirtschaftliche Programme, gleichzeitig mit Nahrungsmittelhilfeaktionen, oder durch Aktionen für eine bessere Funktionsweise der Gefängnis- und Justizeinrichtungen). Schliesslich berücksicht das IKRK bei der Evaluation der Bedürfnisse das wirtschaftliche, soziale und kulturelle Umfeld, auch wenn die Definition des Allernötigsten von einem Kontinent zum anderen unterschiedlich ist. Leiden bleibt ein grundlegendes Kriterium; doch die Art und Weise, wie Leiden empfunden wird, birgt einen Teil Subjektivität in sich.

Das IKRK gedenkt jedoch nicht, alles selbst zu machen. Sein Ziel ist vor allem, die Staaten bzw. andere humanitäre Organisationen, besonders die Nationalen Rotkreuz- und Rothalbmondgesellschaften, zu responsabilisieren, bisweilen den Weg zu zeigen - wie es dies bei Programmen zur Tuberkulosebekämpfung in Gefängnissen getan hat - Unterstützung zu leisten - zum Beispiel durch ein Chirurgenteam in einem staatlichen Krankenhaus - seltener, allein vorzugehen, oder ausnahmsweise, sämtliche nicht befriedigten Bedürfnisse zu decken, wenn es nicht anders geht, vor allem in Haftanstalten.

Stategische Bündnisse mit anderen Partnern entwickeln, besonders mit den Nationalen Rotkreuz- und Rothalbmondgesellschaften und ihrer Föderation

Das IKRK hat sich fest für die Öffnung entschlossen. Es beabsichtigt, seine Erfahrungen und wenn möglich seine Analysen mit anderen zu teilen. Es ist bereit, in bestimmten Situationen die Nothilfeaktionen zu koordinieren. Es möchte seine Bündnisse mit den NRO intensivieren, welche die gleichen Grundsätze einhalten. Ferner hat es beschlossen, eine Strategie von Beziehungen zu bestimmten Kreisen (Wirtschaft, Medien, religiöse Bewegungen und andere nichtstaatliche Akteure) zu errichten. Mit einem Wort, das IKRK will seine diplomatische Tätigkeit im Dienste der Opfer entwickeln. Dies setzt eine ehrgeizige Kommunikationspolitik voraus, die fähig ist, der Stimme des IKRK im Chor der humanitären Debatte Gehör zu verschaffen und eine geschickte Synergie zu erreichen aus einer operationellen Information, die Schlagzeilen in den Medien machen kann, und einer erzieherischen Botschaft, die auf die Achtung der Menschenwürde und der sie schützenden Normen ausgerichtet ist.

Die privilegierten Partner des IKRK sind die Nationalen Rotkreuz- und Rothalbmondgesellschaften und ihre Föderation. Die Gliederung ihrer jeweiligen Verantwortungen wurde in einem Abkommen festgelegt, das im November 1997 in Sevilla angenommen wurde. Dabei handelt es sich um ein Kooperationsabkommen mit dem Ziel, ein Gefühl von Identität, Solidarität, gegenseitigem Vertrauen und gemeinsamer Verantwortung anzuregen. Es ist dem IKRK daran gelegen, alles in seiner Macht stehende zu tun, damit die Internationale Rotkreuz- und Rothalbmondbewegung eine universell bekannte und geachtete eigenständige humanitäre Kraft ist.

\section{$\square$ Die unabhängige humanitäre Aktion, die Kenntnis und Einhaltung} des humanitären Völkerrechts und der humanitären Grundsätze aufwerten

Dieses Ziel entspricht einem Aspekt der Mission des IKRK, der allzu oft verkannt wird: Leiden oder eine Wiederholung der Leiden verursachenden Handlungen zu verhüten. Es ist Zeit für die Institution, alle Gewaltakteure oder jene, die sie beeinflussen können, zum Eintreten für die normativen und ethischen 
Grundsätze, welche die Gewalt in bewaffneten Konflikten einschränken, zu mobilisieren. Dies kann auf vielfache Art erfolgen: indem man zwischen dem humanitären Recht und lokalen Verhaltenskodizes (zum Beispiel zwischen dem humanitären Recht und dem Maya-Recht in Guatemala) Übereinstimmungen sucht, um eine Botschaft auszuarbeiten, welche fähig ist, diejenigen, an die sie sich richtet, $\mathrm{zu}$ interessieren; indem man Mechanismen entwickelt, vorschlägt und zur Anwendung bringt, die geeignet sind, die neuen Gewaltakteure an die humanitären Normen zu binden; indem man ähnliche Kampagnen entwickelt, wie jene, die zum Vertrag von Ottawa über das Verbot von Antipersonenminen führt, oder auch, indem man die von den IKRK-Delegationen lancierten Programme zur Verbreitung des humanitären Völkerrechts intensiviert (vom Harvard-Seminar bis zu Theateraufführungen in Mogadiscio, über Schulprogramme in der Russischen Föderation, Zirkusvorstellungen in Äthiopien und Comics in Ägypten). An Ideen mangelt es nicht. Hierdurch trägt das IKRK zur Ausbildung der Führungskräfte von morgen auf normativer Ebene bei und öffnet sich gegenüber Reflexionen von aussen, die seine Ansichten bereichern, ja sogar verändern können.

Was die Grundsätze der Internationalen Rotkreuz- und Rothalbmondbewegung (Menschlichkeit, Unparteilichkeit, Neutralität, Unabhängigkeit, usw.) betrifft, so sehen die meisten Mitarbeiter des IKRK in ihnen ein wesentliches Merkmal der Institution. Eine wachsende Anzahl von Organisationen berufen sich überdies auf diese Grundsätze, obwohl sie manchmal bei ihrer Anwendung auf Schwierigkeiten stossen. Umso wichtiger ist es, darüber nachzudenken, mit welchen Mitteln man diese Grundsätze besser verständlich machen kann.

\section{$\square$ Die Effizienz der Institution steigern}

Um noch professioneller $\mathrm{zu}$ sein, beabsichtigt das IKRK, seine Verwaltungsmethoden zu verbessern, die Rolle des Hauptsitzes und der Delegationen neu zu definieren, um den Delegationen mehr Autonomie einzuräumen und eine dynamische Personalpolitik zu entwickeln, die einerseits die Förderung der Frauen - eine Stossrichtung, die als von vorrangiger Bedeutung angesehen wird - und andererseits eine optimale Nutzung der oft eindrucksvollen Kompetenzen seiner nationalen Mitarbeiter fördert. Eine besondere Anstrengung soll im Ausbildungsbereich, vor allem bei Planung und Evaluation erfolgen.

Ein Wort zu dem vor sich gehenden Dezentralisierungsprozess: Weit davon entfernt, bedeutungslos zu sein, wird dieser Prozess eine gründliche Reflexion über die Rolle des Hauptsitzes auslösen, besonders was die Festsetzung der Ausrichtungen im Strategie-, Kontroll- und Evaluationsbereich betrifft. Da die Autonomie der Delegationen immer mehr zunimmt, wird die Herausforderung darin bestehen, eine die Vielfalt unseres Planeten achtende Kohärenz im Denken und Handeln aufrechtzuerhalten. Die Auswirkungen dieser Option auf das Gleichgewicht der menschlichen Ressourcen zwischen dem Hauptsitz und dem Feld bleiben zu ermessen. 


\section{SCHLUSSFOLGERUNGEN}

Die Ziele, die sich das IKRK gesetzt hat, entsprechen vor allem einer veränderten Geisteshaltung, die gekennzeichnet ist durch eine grössere Öffnung bei der Lösung der humanitären Probleme wie auch bei den Bündnissen und durch den Willen, bezüglich der Strategien flexibel zu sein, den lokalen Realitäten nahe zu stehen und gleichzeitig bei der Verfechtung von allem, was in irgendeiner Weise das humanitäre Völkerrecht berührt, beharrlich zu sein.

Niemand kann mit Sicherheit voraussagen, ob die Welt eine Übergangsphase durchmacht oder ob das augenscheinliche Chaos, das soviel Leiden, besonders in Afrika, verursacht, andauern wird. Beim schlimmsten Szenario werden die heute identifizierten Herausforderungen in etwa zwanzig Jahren unter dem Einfluss des Bevölkerungswachstums, der Umweltschäden, unangepasster Konsumweisen und einer Deregulierung der Wirtschaft als gering erscheinen und die auf dem Spiel stehenden Fragen ganz anderer Grössenordnung sein ${ }^{6}$. Deshalb ist es angebracht, unverzüglich Verhütungsaktionen einzuleiten, um beunruhigende Entwicklungen zu unterbinden. Jedoch ist es auch wichtig, einen unabhängigen humanitären Bereich zu bewahren, der ein Eingreifen in Notsituationen ermöglicht. In einer Welt, in der ein globalisiertes kulturelles Modell und partikularistische Identitäten nebeneinander bestehen werden, muss das IKRK seinen Weg finden, um von allen anerkannt und akzeptiert zu werden, denn es wird das Sprachrohr für jene Frauen und Männer sein, die in den Konflikten zu leiden haben. 